\title{
Analysis of the efficacy of liquid-based cytology combined with HPV genotypes in screening cervical lesions in women of different ages
}

https://doi.org/10.1515/labmed-2019-0144

Received August 15, 2019; accepted March 26, 2020; previously published online June 4, 2020

\section{Abstract}

Objectives: To evaluate the value of liquid-based cell examination combined with human papillomavirus (HPV) genotypes in the detection of cervical intraepithelial neoplasia.

Methods: A total of 21,155 patients who had undergone cytology, HPV genotypes and colposcopy-guided biopsy were grouped into three groups. Sensitivity, specificity, positive predictive value, and negative predictive value of the test methods of the three groups were compared.

Results: In the age group of less than 50 years, the sensitivity of HPV genotype testing was significantly higher than that of liquid-based cytology. The specificity of liquid-based cytology was significantly higher in all age groups than that of HPV genotype testing. The positive predictive value of each test index was higher in the age group less than 30 years, and the negative predictive value was higher in the 30-50-year age groups. In different age groups, liquid-based cytology and HPV genotype combined method can improve the sensitivity of detection and negative predictive values.

Conclusions: The HPV test has higher sensitivity and negative predictive value, and the cytology method is relatively specific and less sensitive. The combined method has better sensitivity and negative predictive value.

Keywords: biopsy; cervical intraepithelial neoplasia; colposcopy; human papillomavirus; liquid-based cytology.

\footnotetext{
*Correspondence: Chao-Yan Yue, Department of Laboratory Medicine, Fudan University Affiliated Obstetrics and Gynecology Hospital, Shanghai, P.R. China, E-Mail: yuechaoyan@sina.com. https://orcid.org/0000-0003-4030-0166

Jun Jin: Department of Pathology, Fudan University Affiliated Obstetrics and Gynecology Hospital, Shanghai, P.R. China
}

\section{Introduction}

The incidence of cervical cancer has become the second most common malignant tumor in the female reproductive system. In recent years, the incidence of cervical cancer is still on the rise and has a trend of aging, in order to become a serious threat to the health and life of women in China. Cervical cancer is the only malignant tumor with definite carcinogenic etiology at present. The incidence of cervical cancer is related to the persistent infection of high-risk human papillomavirus (HPV) [1, 2]. There are an estimated 570,000 new cases of cervical cancer worldwide in 2018 [3]. When a woman is infected with HPV, she is usually naturally cleared within a year and will not progress to cervical cancer. However, some women infected with the virus cannot eliminate themselves and continue to infect for more than a year, resulting in persistent HPV infection. In this case, HPV infection develops into precancerous lesions of the cervix and eventually into cervical cancer [4-6]. At present, there are three available cervical cancer screening strategies: HPV-based screening strategy, cytology-based screening strategy and naked eye-based screening strategy (acetic acid and/or Lugol iodine). Studies have shown that HPV-DNA-based screening technology is a more effective screening tool for cervical cancer than the other two alternative technologies. At present, the World Health Organization's recommendations for cervical cancer screening are based on HPV screening technology [7]. Persistent high-risk HPV infection is the main cause of cervical cancer. In the initial screening of cervical cancer, HPV genotype testing is more effective than cytology, relatively cheap and easy to operate, so the international guidelines recommend HPV genotype testing as the preferred screening method for cervical cancer $[8,9]$.

Because of the transient infection of HPV, the diagnostic value of different screening methods for women of different ages is different; so our study divides the subjects into groups according to age, in order to analyze the diagnostic efficacy of liquid-based cytological, HPV genotype 
testing and the combination of liquid-based cytology and the two methods, so as to provide a theoretical basis for clinicians in the selection of screening methods for cervical cancer.

\section{Materials and methods}

\section{Subjects}

The patients who underwent cervical biopsy from November 2016 to November 2018 were selected from the Obstetrics and Gynecology Hospital of Fudan University. Patients with a history of cervical surgery were excluded. The most recent liquid-based cytology results, HPV genotype testing results and colposcopy biopsy results of patients in this range were collected for retrospective study. A total of 21,152 cases were included in this study, with an average age of $42 \pm 12$ years, a minimum age of 15 years, a maximum age of 87 years and a median age of 40 years. According to the age group, they were divided into three groups: 3466 cases under 30 years of age, with an average age of $26 \pm 3$ years. Twelve thousand one hundred and fifty-eight cases were more than or equal to 30 years of age and less than 50 years of age, with an average age of $39 \pm 6$ years. Five thousand five hundred and twentyeight patients were more than or equal to 50 years of age (mean age $57 \pm 6$ years). Pathological biopsy was used as the "gold standard" in all patients.

\section{Liquid-based cytology}

A liquid-based cytological technique developed by Biddy Company of the United States was used. The settling liquidbased cytology technology put the whole sampling brush into the cell preservation solution for inspection, which avoided the loss of effective information of the sample to the greatest extent. Cell preservation solution has the function of maintaining cell morphology, transforming mucus and dealing with red blood cells. In the pre-treatment of specimens, the use of cell preservation solution and gradient centrifugation removed a large number of interference components such as blood and mucus that affect diagnosis and improved the quality of cell slices. In this study, sedimentation technique was used as the cytological screening method. According to tris buffered saline criteria, pathological cytological diagnosis can be divided into: no intraepithelial lesions or malignant tumor cells, atypical squamous epithelial cells-unclear significance
(ASC-US), atypical squamous epithelial cells-excluding high-grade squamous intraepithelial lesions (ASC-H), low-grade squamous intraepithelial lesion (LSIL), high squamous intraepithelial lesion (HSIL) and squamous cell carcinoma (SCC), atypical glandular cell, adenocarcinoma and adenosquamous carcinoma. The results were positive except for no intraepithelial lesions or malignant tumor cells as negative reports.

\section{HPV genotype testing}

A complete set of equipment and reagents of Roche's cobas 4800 HPV detection system (Roche Molecular Systems, Pleasanton, CA, USA) was used. The detection principle was multiple-fluorescence polymerase chain reaction (PCR) qualitative testing. The detection results can distinguish HPV16, HPV18 and other 12 high-risk HPV subtypes (HPV 31, 33, 35, 39, 45, 51, 52, 56, 58, 59, 66 and 68) $[10]$.

\section{HPV genotype combined cytological examination}

The HPV results of the same patient were combined with the cytological results, one of which was positive or both of them were positive, which was regarded as the positive result of the combined test, and the negative results of the two tests were recorded as negative results.

\section{Colposcopy-guided biopsy}

The cervix is fully exposed, the secretions are removed and the cervical surface is coated with 3\% acetic acid solution. Colposcopy was used to observe whether the cervical images were satisfactory, whether there were abnormal white epithelium, blood vessels and glandular openings, $1 \%$ iodine solution-coated cervix, and colposcopy to observe whether there was iodine non-staining area. For the above abnormal sites, biopsy forceps were used to bite several pieces of tissue with a diameter of $0.2 \mathrm{~cm}$ as deep as interstitial, and if the lesions of cervical canal could not be ruled out if they were not satisfied with the image, the inner membrane tissue of cervix was scraped with curette. The sampled tissues were stored in $4 \%$ formaldehyde glass bottle for pathological examination and routine paraffin section diagnosis. The positive results of pathological biopsy included low grade, high grade and cancer, and the rest were reported as negative. 


\section{Evaluation index}

The diagnostic results of pathological biopsy were taken as the "gold standard", and cytological examination and HPV test were used as the primary screening results. Compared with pathological biopsy, it can be divided into four types: pathological biopsy positive and primary screening positive as true positive, biopsy positive primary screening negative as false negative, biopsy negative and primary screening negative as true negative, biopsy negative and primary screening negative. The screen positive was false positive. The sensitivity, specificity, positive predictive value and negative predictive value of the three methods were statistically analyzed.

\section{Statistical method}

GraphPad Prism version 5.0 for Windows (GraphPad Software, San Diego, CA, USA) was used for statistical processing, and chi-square $\left(\chi^{2}\right)$ test was used to compare the counting data. The difference was statistically significant $(\mathrm{p}<0.05)$.

\section{Results}

\section{Age $<30$ years}

There were 3466 cases, 1154 cases were cytological positive and 12,654 cases were cytological negative. According to the diagnostic criteria of pathological biopsy, 4113 cases were true positive, 10,803 cases were true negative, 4385 cases were false positive and 1851 cases were false negative. There were 2121 cases of HPV positive and 1345 cases of HPV negative. According to the diagnostic criteria of pathological biopsy, 1028 cases were true positive, 1106 cases were true negative, 1093 cases were false positive and 239 cases were false negative. Two thousand six hundred and fifty-nine cases were positive and 807 cases were HPV negative. According to the diagnostic criteria of pathological biopsy, 1178 cases were true positive, 718 cases were true negative, 1481 cases were false positive and 89 cases were false negative. The sensitivity, specificity, positive predictive value and negative predictive value are detailed in Table 1.

\section{Age $30 \leq$ years $<50$}

There were 12,158 cases, 4433 cases were cytological positive and 7725 cases were cytological negative. According to the diagnostic criteria of pathological biopsy, 2179 cases were true positive, 6700 cases were true negative, 2254 cases were false positive and 1025 cases were false negative. There were 5816 cases of HPV positive and 5611 cases of HPV negative. According to the diagnostic criteria of pathological biopsy, 2473 cases were true positive, 5611 cases were true negative, 3343 cases were false positive and 731 cases were false negative. Seven thousand six hundred and twenty-nine cases were positive and 4529 cases were HPV negative. According to the diagnostic criteria of pathological biopsy, 2953 cases were true positive, 4278 cases were true negative, 4676 cases were false positive and 251 cases were false negative. The sensitivity, specificity, positive predictive value and negative predictive value are detailed in Table 2.

\section{Age $\geq 50$ years}

There were 5528 cases, 2511 cases were cytological positive and 3017 cases were cytological negative. According to the diagnostic criteria of pathological biopsy, 1089 cases were true positive, 2613 cases were true negative, 1422 cases were false positive and 404 cases were false negative. There were 2916 cases of HPV positive and 2612 cases of HPV negative. According to the diagnostic criteria of pathological biopsy, 1130 cases were true positive, 2249 cases were true negative, 1786 cases were false positive and 363 cases were false negative by HPV. Three thousand nine hundred and seventy-three cases were positive and

Table 1: Detection efficiency of liquid-based cytology and HPV-DNA in patients under 30 years of age.

\begin{tabular}{lrrrr}
\hline Groups & Sensitivity, \% & Specificity, \% & Positive predictive value, \% & Negative predictive value, \% \\
\hline Liquid-based cytology & 66.69 & 67.76 & 54.38 & 77.93 \\
HPV-DNA detection & 81.14 & 50.30 & 48.47 & 82.23 \\
Joint detection & 92.28 & 32.65 & 44.30 & 88.97 \\
$\chi^{2}$ & 277.200 & 542.067 & 39.978 & 46.610 \\
p-Value & 0.000 & 0.000 & 0.000 & 0.000 \\
\hline
\end{tabular}


Table 2: Detection efficiency of liquid-based cytology and HPV-DNA in the age group 30-50 years.

\begin{tabular}{lrrrr}
\hline Groups & Sensitivity, \% & Specificity, \% & Positive predictive value, \% & Negative predictive value, \% \\
\hline Liquid-based cytology & 68.01 & 74.83 & 49.15 & 86.73 \\
HPV-DNA detection & 77.18 & 62.66 & 42.52 & 88.47 \\
Joint detection & 92.17 & 47.78 & 38.71 & 94.46 \\
$\chi^{2}$ & 576.794 & 1391.641 & 125.180 & 182.418 \\
p-Value & 0.000 & 0.000 & 0.000 & 0.000 \\
\hline
\end{tabular}

Table 3: Detection efficiency of liquid-based cytology and HPV-DNA in the age group greater than or equal to 50 years.

\begin{tabular}{lrrrr}
\hline Groups & Sensitivity, \% & Specificity, \% & Positive predictive value, \% & Negative predictive value, \% \\
\hline Liquid-based cytology & 72.94 & 64.76 & 43.37 & 86.61 \\
HPV-DNA detection & 75.69 & 55.74 & 38.75 & 86.10 \\
Joint detection & 92.30 & 35.69 & 34.68 & 92.60 \\
$\chi^{2}$ & 207.119 & 715.931 & 49.550 & 44.248 \\
p-Value & 0.000 & 0.000 & 0.000 & 0.000 \\
\hline
\end{tabular}

1555 cases were HPV negative. According to the diagnostic criteria of pathological biopsy, 1378 cases were true positive, 1440 cases were true negative, 2595 cases were false positive and 115 cases were false negative. The sensitivity, specificity, positive predictive value and negative predictive value are detailed in Table 3.

\section{Discussion}

Cervical cancer has occupied the fourth place in the cause of death of female malignant tumors. More and more attention has been paid to the prevention and screening of cervical cancer and the screening methods are also constantly updated and developed. From the initial Pap smear to the subsequent use of liquid-based cytology, as well as in the past decade, HPV has become a more mainstream auxiliary screening method in China. Pap smear is the earliest auxiliary method used in clinic, because the quality of Pap smear is affected by various interference factors, such as material location, smear mode, blood smear, inflammatory cells and so on. Its sensitivity and specificity are not high [11], many areas have been completely replaced by liquid-based cytology. In this study, the sensitivity of cytology increased with age. However, liquid-based cytology has some limitations due to the lack of histological characteristics. Due to subjective reasons such as sampling and morphological observation, misdiagnosis will inevitably occur [12].

HPV has been recognized as the main cause of cervical lesions in women. About $70 \%$ of cervical precancerous lesions and cervical cancer are caused by subtypes 16 and 18. However, not all women infected with HPV develop cervical lesions, and $80 \%$ of women are likely to be infected with HPV in their lifetime [13]. Studies have shown that HPV nucleic acid testing is more sensitive than cytology, but slightly less specific, because most HPV infections are asymptomatic and disappear spontaneously. HPV nucleic acid testing is more objective, more direct and easier to automate, while the diagnostic value of cytology is affected by strict pre-analysis requirements and observer skills. Because cytology is prone to false-negative results, HPV genotypes can be used as an attractive alternative for screening of cervical cancer [14].The Society of Cervical Pathology's (ASCCP) guidelines for screening cervical lesions, which included HPV as an auxiliary cytology method in 2006, and the 2012 edition recommend combined cytological and HPV screening for women over 30 and 65 years of age [15], and for younger women aged 21 and 29 years, cytology is recommended for screening.

Compared with cytology, HPV has higher sensitivity and negative predictive value. In this study, the sensitivity of HPV genotypes is higher than that of liquid-based cytology, and the difference is statistically significant, which reduces the possibility of missed diagnosis. But at the same time, compared with cytology, the specificity of the HPV genotype test was lower than that of liquid-based cytology, and the difference was statistically significant, which indicated that the possibility of misdiagnosis of HPV alone as a screening method was higher than that of cytological test. It was verified that there were a large number of transient infections in female patients with 
HPV infection. When young women are infected with the virus, they cannot clear the virus and enter the stage of persistent infection. It is of great significance to detect HPV in older women or women who have had sex for a long time. In this study, the positive predictive value of HPV in different age groups was significantly different from $48.47 \%$ under 30 years of age to $38.75 \%$ over 50 years of age. With the increase of age group, it showed a trend of gradual decrease. It may be related to the local immunity status of the young population and the lack of sexual health knowledge and other factors.

From the comparison of independent data of liquidbased cytology and HPV genotype test, it can be seen that cytology has relatively high specificity and low sensitivity. On the contrary, HPV test, the combination of the two will take advantage of each other. From this data analysis, the sensitivity of cytological and HPV genotype testing combined methods was as high as $92 \%$, which was significantly different from that of independent screening $(\mathrm{p}<0.05)$, which greatly reduced the possibility of missed diagnosis. In the aspect of specificity, the specificity of HPV test was lower than that of single cytological test $(\mathrm{p}<0.05)$, and the misdiagnosis was greatly increased. This phenomenon was still related to the transient infection of HPV, at the same time. Combined methods showed that compared with the independent use of HPV and cells, the negative predictive value was increased and the misdiagnosis rate was reduced.

Recently, it has been reported that the abnormal rate of liquid-based cytology increases with the increase of female age, while the positive rate of high-risk HPV shows a bimodal age model. Therefore, there are limitations to only HPV testing in young women, because a high proportion of HPV infections in young women will be automatically cleared. Because of the low specificity of HPV-based screening, it is not applied to women under the age of 30 years and certain populations under the age of 35 years $[16,17]$. A study from China points out that age is an indicator for identifying high-risk patients in HPV-positive ASCUS patients. The risk of cervical intraepithelial neoplasia $3+$ is significantly increased in HPVpositive ASCUS patients over 60 years of age, so clinicians should pay more attention to it [3]. A Swedish study found that the most common hrHPV genotypes in women aged 55-59 years are non-HPV-16/18 genotypes, which represent most histologically confirmed HSIL lesions in this population. Cytological classification after HPV positive showed no additional benefit in this population. HPVbased screening has the potential to prevent additional cases of cervical cancer in women over 60 years of age [18]. Although it is generally believed that HPV screening under the age of 30 years is not desirable, due to the lack of empirical evidence, there is still no consensus on the age of stopping screening. A recent simulation study estimated the remaining lifetime risk of cervical cancer with different exit ages and different exit tests. The results show that cytological screening under the age of 75 years can prevent cervical cancer in later years, and women who are negative for HPV screening after the age of 55 years have a low risk of developing cervical cancer for the rest of their lives [19].

To sum up, the HPV genotype test has high sensitivity and high negative predictive value, while HPV genotype detection has a high degree of automation and standard quality control rules. The method of cytological examination has high specificity, but low sensitivity, and the interpretation of the results in this method is subjective and requires high professional level of doctors. However, because of the high sensitivity of the HPV genotype test, the results contain a large number of "transient" infections, which may increase the possibility of overtreatment. In view of the limitations of HPV testing, it is necessary to formulate a further diagnosis and treatment plan for women with a high risk of HPV DNA according to the age of the patients. Cytology is the first choice for HPV screening positive. With the help of the characteristics of high specificity of cytology, it can neutralize the high sensitivity and low positive predictive value of the HPV genotype test, and reduce the referral rate of colposcopy. It has a significant advantage in the screening of a large sample population. The combined method has good sensitivity and negative predictive value, can effectively avoid missed diagnosis and misdiagnosis and has good clinical application prospects.

There are also some limitations in our study. Among our subjects, there is no distinction between whether they have been vaccinated against HPV or not. This is a retrospective study conducted in a single institution and may have a selection bias.

In conclusion, when selecting the screening method for cervical intraepithelial neoplasia, the age of the patient should be taken into account. Liquid-based cytology is recommended as a screening method in the age group under 30 years, and the combined method of liquid-based cytology and HPV genotypes is recommended in the age group from 30 to 50 years.

Research funding: None declared.

Author contributions: All authors have accepted responsibility for the entire content of this manuscript and approved its submission. 
Competing interests: Authors state no conflict of interest. Informed consent: Informed consent was obtained from all individuals included in this study.

Ethical approval: Research involving human subjects complied with all relevant national regulations, institutional policies and is in accordance with the tenets of the Helsinki Declaration (as revised in 2013). Approval was obtained from the Research Ethics Committee of the Obstetrics \& Gynecology Hospital of Fudan University (approval number: 2019-06).

\section{References}

1. Trottier H, Burchell AN. Epidemiology of mucosal human papillomavirus infection and associated diseases. Public Health Genom 2015;18:291-307.

2. Ferlay J, Soerjomataram I, Dikshit R, Eser S, Mathers C, Rebelo $M$, et al. Cancer incidence and mortality worldwide: sources, methods and major patterns in GLOBOCAN 2012. Int J Cancer 2015;136:E359-86.

3. Wang YY, Kong LH, Liu Y, Wang S, Fan QB, Zhu L, et al. Retrospective analysis of cervical cancer and precancerous lesions in patients with atypical squamous cells of undetermined significance in China. Medicine (Baltimore) 2019;98:e18239.

4. Song SH, Lee JK, Seok OS, Saw HS. The relationship between cytokines and HPV-16, HPV-16 E6, E7, and high-risk HPV viral load in the uterine cervix. Gynecol Oncol 2007;104:732-8.

5. Tjiong MY, Out TA, Ter Schegget J, Burger MP, Van Der Vange N. Epidemiologic and mueosal immunologic aspects of HPV infection and HPV-related cervical neop lasiain the lower female genital tract: a review. Int J Gyneeol Cancer 2001;11: 9-17.

6. Ali MA, Bedair RN, Abd El Atti RM. Cervical high-risk human papillomavirus infection among women residing in the Gulf Cooperation Council countries: prevalence, type-specific distribution, and correlation with cervical cytology. Cancer Cytopathol 2019;127:567-77.

7. Ndizeye Z, Menon S, Van Geertruyden JP, Sauvaget C, Jacquemyn Y, Bogers JP, et al. Performance of OncoE6TM Cervical Test in detecting cervical precancer lesions in HIV-positive women attending an HIV clinic in Bujumbura, Burundi: a cross-sectional study. BMJ Open 2019;9:e029088.
8. Gustavsson I, Aarnio R, Myrnäs M, Hedlund-Lindberg J, Taku 0 , Meiring T, et al. Clinical validation of the HPVIR high-risk HPV test on cervical samples according to the international guidelines for human papillomavirus DNA test requirements for cervical cancer screening. Virol J 2019;16:107.

9. Li L, Zheng Z, Li L. Evaluation of human-papillomavirus screening for cervical cancer in China's rural population. Peerl 2019;7:e8152.

10. Tao X, Zhang H, Li J, Zhang H, Xiao J, Zhang L, et al. Prevalence of HPV-16/18 genotypes and immediate histopathologic correlation results in a Chinese population with negative cytology and positive high-risk HPV testing. Cancer Cytopathol 2019;127:650-7.

11. Nanda K, McCrory DC, Myers ER, Bastian LA, Hasselblad V, Hickey JD, et al. Accuracy of the Papanicolaou test in screening for and follow-up of cervical cytologic abnormalities: a systematic review. Ann Intern Med 2000;132:810-9.

12. Wei-Tong H, Hong-Wei W, Wei H, Hui-Zhen W, Zheng-Yi C, Ying-Qun W. Combined application of liquid based cytology and pathogenic microbes in the detection of cervical cancer. Chin J Lab Diagn 2004;8:507-9.

13. Dunne EF, Unger ER, Sternberg M, McQuillan G, Swan DC, Patel SS, et al. Prevalence of HPV infection among females in the United States. J Am Med Assoc 2007;297:813-9.

14. Levi JE, Martins TR, Longatto-Filho A, Cohen DD, Cury L, Fuza $L M$, et al. High-risk HPV testing in primary screening for cervical cancer in the public health system, São Paulo, Brazil. Cancer Prev Res (Phila) 2019;12:539-46.

15. Saslow D, Solomon D, Lawson HW, Killackey M, Kulasingam SL, Cain J, et al. American Cancer Society, American Society for Colposcopy and Cervical Pathology, and American Society for Clinical Pathology screening guidelines for the prevention and early detection of cervical cancer. J Low Genit Tract Dis 2012;16:175-204.

16. Şuteu 0 , Blaga ML, Nygård M, Leinonen MK, Nicula F, Păiş R, et al. Prevalence of positive screening test results and agreement between cytology and human papillomavirus testing in primary cervical cancer screening in North-Western Romania. Eur J Cancer Prev 2020;29:141-8.

17. Maver PJ, Poljak M. Primary HPV-based cervical cancer screening in Europe: implementation status, challenges, and future plans. Clin Microbiol Infect 2020;26:579-83.

18. Bergengren L, Lillsunde-Larsson G, Helenius G, Karlsson MG. HPV-based screening for cervical cancer among women 55-59 years of age. PLoS One 2019;14:e0217108.

19. Malagón T, Kulasingam S, Mayrand MH, Ogilvie G, Smith L, Bouchard C, et al. Age at last screeningand remaining lifetime risk of cervical cancer in older, unvaccinated, HPV-negative women: a modelling study. Lancet Oncol 2018;19:1569-78. 